\title{
Functional analysis of Drosophila transcription factor IIB
}

\author{
Sharon L. Wampler and James T. Kadonaga ${ }^{1}$ \\ Department of Biology and Center for Molecular Genetics, University of California, San Diego, \\ La Jolla, California 92093 USA
}

We have isolated a cDNA encoding Drosophila transcription factor IIB (dTFIIB) and characterized the properties of recombinant dTFIB with a reconstituted in vitro transcription system derived from Drosophila embryos. Purified, recombinant dTFIIB is fully active at a concentration of one molecule per template DNA. With different promoters, the transcriptional activity of dTFIB was similar but not identical to that of human TFIIB, which suggests that there may be variations in the mechanisms by which TFIIB functions in transcription. We have also found that recombinant dTFIIB suppressed nonspecific initiation of transcription by RNA polymerase II by a mechanism that appears to involve direct interaction between TFIIB and the polymerase. Addition of excess dTFIIB to transcription reactions resulted in promoter-specific repression of transcription. These experiments have led to the hypothesis that TFIIB interacts with a basal transcription factor that is required for transcription of some, but not all, genes and that the presence of excess dTFIIB results in sequestration of the promoter-specific basal factor to prevent its assembly into a productive transcription complex. Excess dTFIIB did not, however, affect the ability of either GAL4-VP16 or Sp1 to stimulate transcription. These data indicate that in contrast to current models, GAL4 derivatives do not activate transcription by increasing the rate of assembly of TFIIB into the transcription complex.

[Key Words: Drosophila TFIIB gene; RNA polymerase II; in vitro transcription; basal transcription factors; squelching]

Received April 7, 1992; revised version accepted May 20, 1992.

Synthesis of mRNA is a fundamental and important process that has a central role in the expression of genes. In eukaryotes, basal transcription of protein-coding genes is carried out by RNA polymerase II and several auxiliary factors that are sometimes referred to as the general factors (for recent review, see Saltzman and Weinmann 1989; Sawadogo and Sentenac 1990; Conaway and Conaway 1991; Zawel and Reinberg 1992). Transcription by the basal machinery is affected by promoter- and enhancer-binding factors (for review, see Johnson and McKnight 1989; Mitchell and Tjian 1989/ that appear to act in conjuction with another class of factors known as coactivators, mediators, adapters, or intermediary factors (see, e.g., Ptashne and Gann 1990; Dynlacht et al. 1991; Flanagan et al. 1991; Pugh and Tjian 1992). The chromatin structure of the template DNA is also an important determinant of transcriptional activity (for recent review, see Wolffe 1990; Kornberg and Lorch 1991; Felsenfeld 1992). Hence, regulation of transcription by RNA polymerase II minimally involves the basal transcription apparatus, sequence-specific DNA-binding factors, coactivators/mediators, and the chromatin template.

The mechanism of basal transcription by RNA polymerase II has been examined by fractionation and puri-

${ }^{1}$ Corresponding author. fication of RNA polymerase II and the general factors. These studies have been carried out with transcription factors from HeLa cells, rat liver, Drosophila, and Saccharomyces cerevisiae (for review, see Saltzman and Weinmann 1989; Sawadogo and Sentenac 1990; Conaway and Conaway 1991; Zawel and Reinberg 1992). There have been many advantages to this diversity in the biochemical analysis of RNA polymerase II transcription. The transcription process is somewhat complex and is particularly susceptible to a variety of artifacts, and the comparison of fractionated transcription systems derived from different organisms has led to significant insights into the mechanism of transcription. Nevertheless, many fundamental aspects of basal transcription remain to be clarified. For instance, the identity of each of the general factors that are required for basal transcription is not yet known. There are also other facets of the transcription process, such as the biochemical functions of the general factors, that need to be illuminated.

We have been studying the biochemistry of RNA polymerase II transcription in Drosophila by using embryos as the source of transcription factors. The Drosophila embryo transcription system has high transcriptional activity (Kadonaga 1990; Kamakaka et al. 1991), and the fractionated general factors from Drosophila embryos 
are functionally interchangeable with their human counterparts (Wampler et al. 1990). Furthermore, Drosophila embryos are relatively inexpensive and can be obtained in kilogram quantities. The Drosophila embryo transcription system has been useful for the analysis of multiple rounds of transcription (Kadonaga 1990); studies of in vitro transcriptional activation by the glucocorticoid receptor (Freedman et al. 1989); in vitro transcription of a wide spectrum of genes, including a U1 snRNA gene (Kamakaka et al. 1991); examination of the roles of histone $\mathrm{Hl}$ and nucleosomal cores upon transcriptional regulation (Becker et al. 1991; Croston et al. 1991; Kerrigan et al. 1991; Laybourn and Kadonaga 1991; and the discovery and purification of transcriptional coactivators (Hoey et al. 1990; Pugh and Tjian 1990, 1992; Dynlacht et al. 1991).

In this study we have focused on the function of transcription factor IIB (TFIIB) from Drosophila, which we have designated as dTFIIB to distinguish the Drosophila transcription factor from its human counterpart, hTFIIB. TFIIB has been purified from HeLa cells (Reinberg and Roeder 1987; Maldonado et al. 1990; Ha et al. 1991; Moncollin et al. 1992) and rat liver (Conaway et al. 1987), and the gene encoding human TFIIB has been isolated (Ha et al. 1991). In addition, a gene that encodes a TFIIB homolog in $S$. cerevisiae (yTFIIB) has been described (Pinto et al. 1992). TFIIB appears to assemble into the transcription preinitiation complex at an early stage after the binding of TFIID and TFIIA to the template DNA (Buratowski et al. 1989; Maldonado et al. 1990). The yeast homolog of TFIIB appears to be required for start site selection (Pinto et al. 1992). Studies of transcriptional activation by GAL4 derivatives, such as GAL4AH and GAL4-VP16 hybrid proteins, suggest that the GAL4 derivatives activate transcription by recruiting TFIIB to the promoter by direct binding of TFIIB to the GAL4 derivative (Lin and Green 1991; Lin et al. 1991; for review, see Hawley 1991; Sharp 1991). Previously, we have subdivided the RNA polymerase II machinery from Drosophila embryos to give a fraction containing dTFIIB activity as well as a TFIIB-dependent transcription system (Wampler et al. 1990). In this study we describe the isolation of the Drosophila gene encoding TFIIB and the functional characterization of recombinant dTFIIB in the context of the transcription reaction.

\section{Results}

\section{Isolation of the Drosophila TFIIB gene}

We have isolated a cDNA encoding the Drosophila version of TFIIB from a Drosophila embryo cDNA library in $\lambda \mathrm{gt} 10$ by using the human TFIIB gene /a generous gift of Ilho Ha and Dr. Danny Reinberg, University of Medicine and Dentistry of New Jersey, Piscatawayl as a probe. The nucleotide sequence and predicted amino acid sequence of dTFIIB is given in Figure 1A. The Drosophila TFIIB gene encodes a protein containing 315 amino acid residues with a calculated molecular mass of 34,347 daltons. A comparison of the predicted amino acid sequences of
TFIIB from Drosophila, human, and yeast is shown in Figure 1B. There is $79 \%$ amino acid identity between dTFIIB and hTFIIB. This high degree of conservation of primary amino acid sequence is consistent with the previous demonstration that ATFIIB and hTFIIB are functionally interchangeable in reconstituted transcription reactions (Wampler et al. 1990) and cross-react by Western blot analysis with antibodies against hTFIIB /data not shown). Northern blot analysis with Drosophila embryo RNA revealed a major transcript of $1.5 \mathrm{~kb}$, which corresponds roughly with the length of the cDNA, and a minor transcript of $1.3 \mathrm{~kb}$ (data not shown). Southern blot analysis indicated that dTFIIB is encoded by a single gene (data not shown). By in situ hybridization to polytene chromosomes, the Drosophila TFIIB locus was mapped to region $31 \mathrm{~F}$ on the left arm of the second chromosome (J. Lis, unpubl.). Structural motifs of TFIIB that have been noted previously in hTFIIB and yTFIIB, such as a putative amphipathic helix and a potential $\mathrm{Zn}$ (II) finger (Ha et al. 1991; Pinto et al. 1992), are also present in dTFIIB.

Transcriptional activity of purified, Escherichia coli-synthesized dTFIIB

To characterize the biochemical properties of dTFIIB, it was first necessary to express and to purify the protein and then to test the transcriptional activity of the recombinant dTFIIB in a reconstituted transcription reaction. To this end, we modified the DNA sequence at the methionine initiation codon to a NdeI site by site-directed mutagenesis and subcloned the TFIIB-coding sequence into the pET11a E. coli expression vector (Studier et al. 1990). dTFIIB was expressed efficiently in E. coli, as shown by the appearance of a $34.5-\mathrm{kD}$ band upon induction of $E$. coli cells containing the Drosophila TFIIB gene (Fig. 2A, cf. lanes 2 and 3). The dTFIIB protein was purified from the crude extract to $>95 \%$ homogeneity by chromatography on phosphocellulose and hydroxylapatite resins (Fig. 2A, lanes 4,5). We examined the ability of the purified, recombinant dTFIIB to function in the fractionated transcription system from Drosophila embryos (Wampler et al. 1990). In the absence of TFIIB, there was no detectable transcription (Fig. 2B, lane 1). Upon addition of native dTFIIB from Drosophila embryos, recombinant dTFIIB, or $\alpha$, a rat liver homolog of TFIIB (gift of Drs. J.W. and R.C. Conaway, Oklahoma Medical Research Foundation), transcription was restored (Fig. 2B, lanes 2-4). Transcription in the presence of recombinant dTFIIB was inhibited by $4 \mu \mathrm{g} / \mathrm{ml}$ of $\alpha$-amanitin, which indicates that transcription was due to RNA polymerase II (Fig. 2B, lane 5). Thus, the purified, recombinant dTFIIB was active for transcription in vitro. Furthermore, in the experiment shown in Figure 2B, the recombinant dTFIIB was active at a concentration of two molecules per $3.4 \mathrm{kbp}$ of template DNA. We have observed additionally that the maximal transcriptional activity of purified, recombinant dTFIIB was sustained to one molecule per template DNA; but at concentrations lower than one molecule per template, the level of transcription decreased (data not shown). These experiments de- 
A

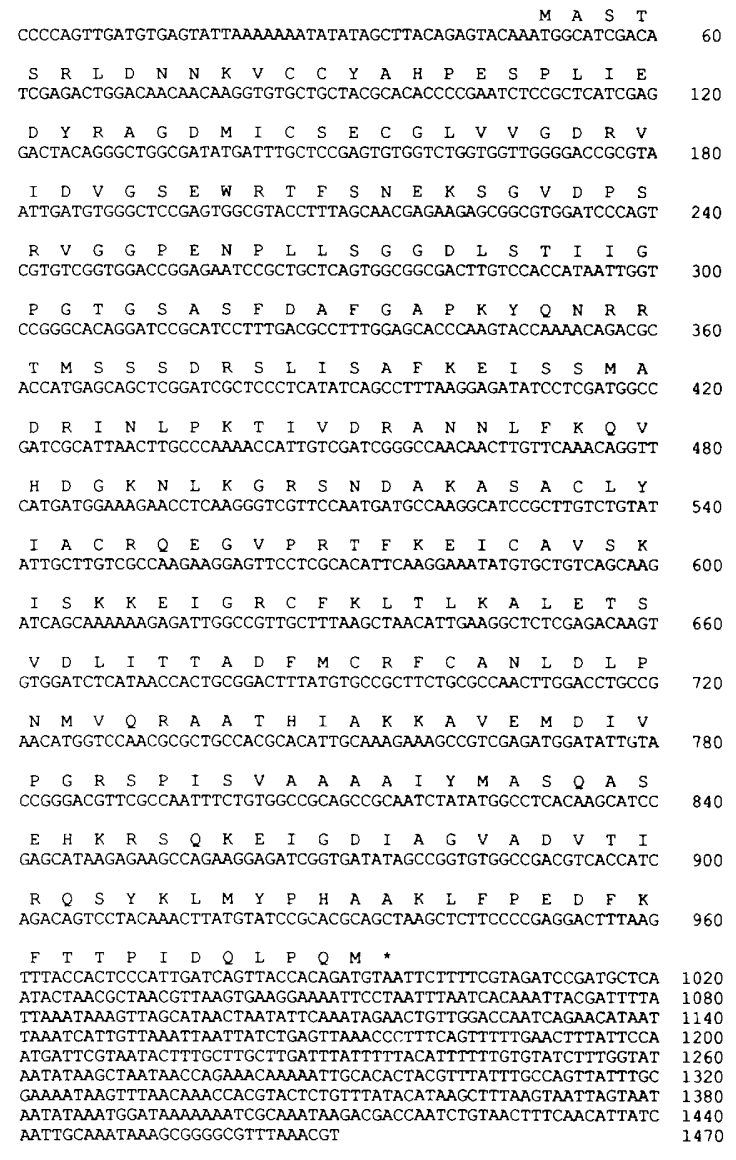

B

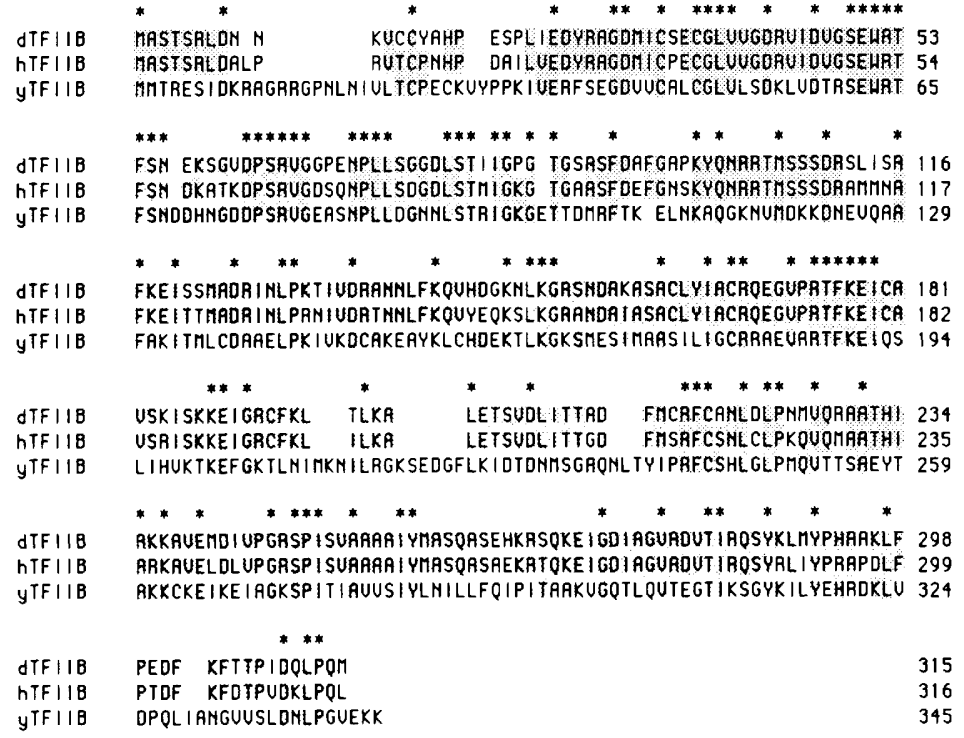

Figure 1. TFIIB from Drosophila. $(A)$ Nucleotide sequence of a cDNA encoding dTFIIB. The predicted amino acid sequence is given in the singleletter amino acid code. The DNA sequence of the dTFIIB cDNA is in GenBank (accession no. M91081). (B) Alignment of the amino acid sequences of TFIIB from Drosophila, human (Ha et al. 1991), and S. cerevisiae (yTFIIB) (Pinto et al. 1992). The analysis of sequence homology was performed by using Doolittle NEWAT protein programs. The conserved amino acid residues are shaded. The asterisks (*) denote positions in which the amino acid residues are identical in all three proteins. monstrated that the purified, recombinant dTFIIB was active for in vitro transcription, and the efficiency of transcription with the recombinant protein suggested that it was predominantly in a transcriptionally active form.

\section{Basal transcription with dTFIIB vs. hTFIIB is similar, but not identical}

Because dTFIIB and hTFIIB are natural variants of TFIIB, we compared the basal transcriptional properties of the two factors with several different promoters. In these studies we employed the following minimal promoters: the Drosophila alcohol dehydrogenase (Adh) proximal promoter $(-56$ to +383 relative to the start site; Benyajati et al. 1983; Heberlein et al. 1985); the Drosophila Krüppel promoter $\mid-31$ to +13 relative to the start site; Rosenberg et al. 1986; Kerrigan et al. 1991); the Drosophila jockey promoter $(-7$ to +343 relative to the start site; Mizrokhi et al. 1988; Wampler et al. 1990); and the adenovirus $\mathrm{E} 4$ promoter $(-38$ to +250 relative to the start site; Lin et al. 1988|. Reconstituted transcription reactions were carried out in the absence of added TFIIB (Fig. 3, lane 1) or in the presence of equivalent amounts (5 ng) of either dTFIIB (Fig. 3, lane 2) or hTFIIB (Fig. 3, lane 4). Where indicated, $\alpha$-amanitin was included in the reactions to confirm that transcription was carried out by RNA polymerase II (Fig. 3, lanes 3,5). dTFIIB and hTFIIB yielded comparable transcriptional levels with the Adh, jockey, and E4 promoters, whereas with the Krüppel promoter, the transcriptional activity of dTFIIB was higher than that of hTFIIB. These transcription reactions were performed with the different promoters in parallel under identical conditions; thus, the differences in the properties of dTFIIB and hTFIIB could not be explained by variability in the activities of the TFIIB preparations. Moreover, the higher level of transcription from the Krüppel minimal promoter with dTFIIB than with hTFIIB was also observed with a larger segment of the Krüppel transcriptional control region that encompasses -861 to +426 relative to the RNA start site (data not shown). This larger segment of the Krüppel promoter is transcribed in vitro about seven times more efficiently than the Krüppel minimal promoter (Kerrigan et al. 1991) and is a stronger promoter in vitro than the Adh, jockey, or adenovirus E4 promoters; hence, the observed preference of the Krüppel promoter variants for dTFIIB does not appear to be related to the efficiency of transcription. A simple interpretation of this effect is that the specific interactions between TFIIB and the other basal factors and the template DNA may vary somewhat at different promoters. 


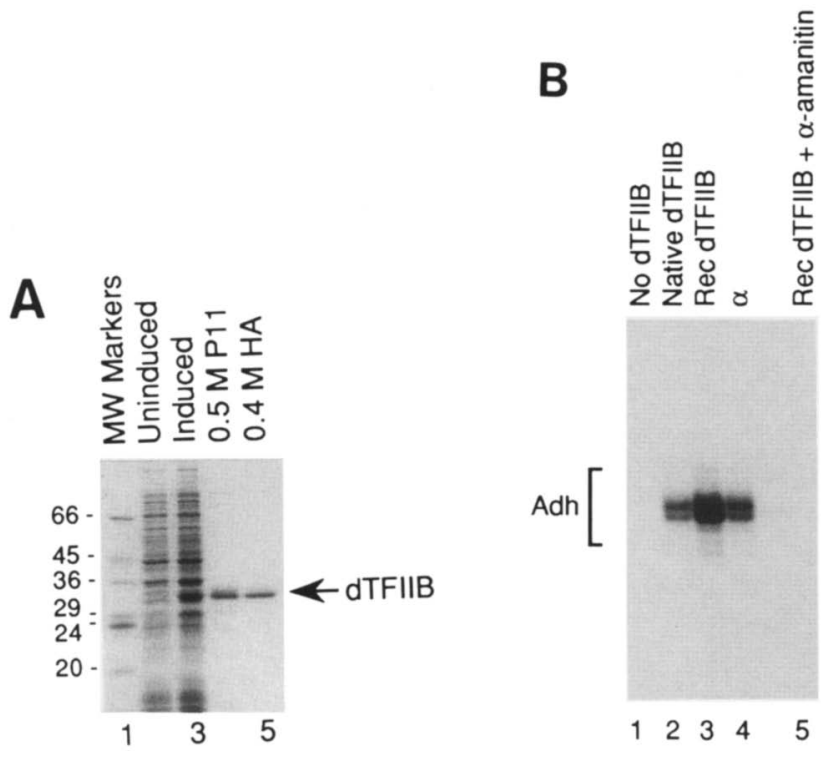

Figure 2. Recombinant dTFIIB expressed in E. coli is active in a reconstituted Drosophila transcription system. (A) Purification of $E$. coli-synthesized dTFIIB. The purity of the recombinant protein was analyzed by $12 \%$ polyacrylamide-SDS gel electrophoresis and staining with Coomassie blue. dTFIIB expressed in E. coli was purified by chromatography on phosphocellulose (P11) and hydroxylapatite (HA) resins. (Lane 1) Molecular mass standards $(1.0 \mu \mathrm{g})$; (lane 2) crude extract of cells in which dTFIIB expression was not induced; (lane 3) crude extract of induced cells; (lane 4) eluate from phosphocellulose column $(0.4 \mu \mathrm{g})$; (lane 5) eluate from hydroxylapatite column $(0.3 \mu \mathrm{g})$. The purified dTFIIB obtained after hydroxylapatite chromatography was used in all subsequent transcription reactions. The sizes of the molecular mass markers are given in kilodaltons. $|B|$ In vitro transcription analysis. The Adh proximal promoter (150 ng of template DNA) was transcribed with a fractionated Drosophila embryo transcription system (Wampler et al. 1990). (Lane 1) No added TFIIB; (lane 2) native dTFIIB (12 ng); (lane 3) recombinant dTFIIB (5 ng); (lane 4) $\alpha$ (rat liver homolog of TFIIB; Conaway et al. 1987); (lane 5) recombinant dTFIIB in the presence of $4 \mu \mathrm{g} / \mathrm{ml}$ of $\alpha$-amanitin. The primer extension products of the Adh transcripts are denoted by brackets.

\section{Purified dTFIIB can suppress nonspecific initiation of transcription by RNA polymerase II}

To investigate potential functional interactions between TFIIB and RNA polymerase II, we examined the effect of dTFIIB on nonspecific transcription initiation by RNA polymerase II. In these experiments we sought to test the effect of dTFIIB on a transcriptional function of polymerase. RNA polymerase II from Drosophila embryos, which was $>90 \%$ homogeneous with no apparent contaminating polypeptides (Wampler et al. 1990), initiated transcription nonspecifically from a template DNA in a manner that was inhibited by $4 \mu \mathrm{g} / \mathrm{ml}$ of $\alpha$-amanitin (Fig. 4, cf. lanes 1, 2, and 5). This reaction occurred at a concentration of two molecules of polymerase per 3.4 $\mathrm{kbp}$ of plasmid DNA (Fig. 4, lane 2). Addition of purified, recombinant dTFIIB at a concentration of either two molecules of dTFIIB per template DNA (Fig. 4, lane 3) or
12 molecules of dTFIIB per template DNA (Fig. 4, lane 4) resulted in a significant decrease in the nonspecific initiation of transcription by the polymerase. Because there were only three purified components in these reactions - the polymerase, dTFIIB, and the template DNA, the suppression of nonspecific transcription initiation was probably due either to direct interactions between dTFIIB and polymerase or to an indirect dTFIIB-mediated repression by a mechanism that involves interactions between dTFIIB and the template DNA, such as nonspecific binding of dTFIIB to the DNA in a manner that prevents access of polymerase to the template. The latter possibility appeared to be unlikely, however, because efficient dTFIIB-mediated suppression was observed at a concentration of only two molecules of dTFIIB per 3.4 kbp of template DNA, which also corresponded to a $1: 1$ molar ratio of dTFIIB to polymerase (Fig. 4, cf. lanes 2 and 3). Hence, it is likely that dTFIIB suppresses nonspecific initiation by RNA polymerase II by direct interactions with the polymerase. Because the polymerase was not $100 \%$ pure, it was also formally possible that dTFIIB suppressed nonspecific transcription by interaction with intermediary factor that binds to polymerase and is present in our preparations of RNA polymerase II. Notwithstanding, in either instance, with or without the extra intermediary factor, it appears that dTFIIB can suppress nonspecific transcription by either a direct or an indirect mechanism.

\section{Promoter-specific squelching of basal transcription by excess dTFIIB}

In the analysis of promoter- and enhancer-binding factors, the presence of abnormally high levels of sequencespecific activators can sometimes lead to an inhibition of transcription, which is popularly known as squelching (Gill and Ptashne 1988; Ptashne 1988; Ptashne and Gann 1990). Squelching appears to be the result of nonproductive interactions between a sequence-specific transcription factor, which is present in excess and is predominantly free in solution, and either a basal transcription factor or a coactivator/mediator. Despite its nonphysiological nature, squelching has provided useful insight into the process of transcriptional activation. In our studies of basal transcription, we similarly sought to examine the effects of excess, purified dTFIIB upon transcription from different promoters. A few possible outcomes of such experiments are as follows. First, if TFIIB were a limiting factor in transcription, then the addition of TFIIB to the reaction would result in an increase in transcription. It is also possible that TFIIB may interact strongly with another essential component in the transcription process and that excess TFIIB, which would be predominantly in solution rather than associated with a preinitiation complex, would squelch transcription by sequestering the other essential component away from the transcription complex. Alternatively, excess TFIIB may have no effect on the efficiency of transcription.

To investigate whether squelching by a basal transcription factor can occur, we performed transcription 
Figure 3. Recombinant dTFIIB and hTFIIB display similar, but not identical, activity with several promoters. Transcription reactions were carried out with the fractionated Drosophila embryo transcription system (Wampler et al. 1990) with the indicated template DNAs (150 ng each). (Lane 1) No added TFIIB; (lane 2) purified, recombinant dTFIIB (5 ng); (lane 3) dTFIIB /5 $\mathrm{ng})+\alpha$-amanitin $(4 \mu \mathrm{g} / \mathrm{ml})$; (lane 4) purified, recombinant hTFIIB $(5 \mathrm{ng})$; (lane 5$)$ hTFIIB $(5 \mathrm{ng})+\alpha$-amanitin $(4 \mu \mathrm{g} / \mathrm{ml})$. The reverse transcription products of the various RNAs are designated by brackets.

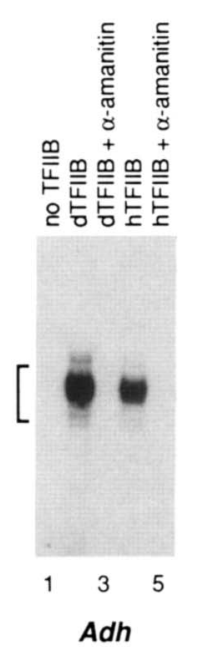

Adh
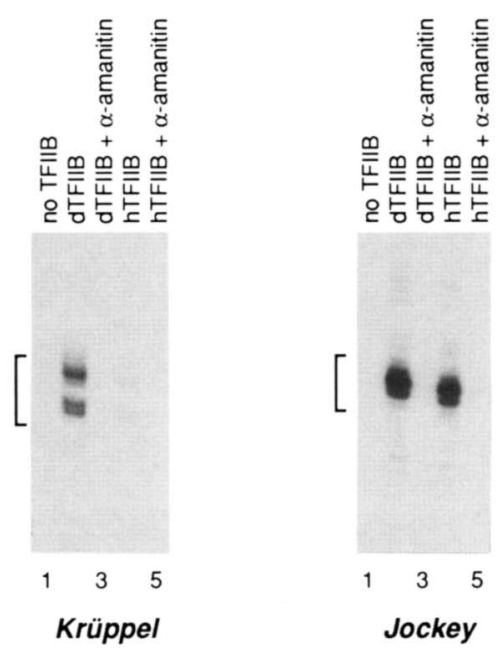

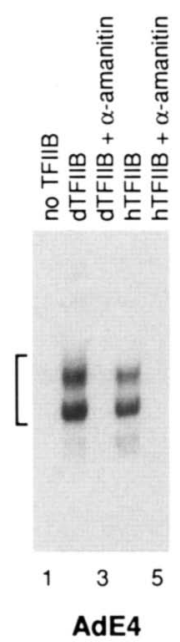

experiments in the presence of excess dTFIIB with the same minimal promoters that were employed in the previous experiments (Fig. 3) with dTFIIB and hTFIIB. In these studies we performed standard transcription reactions with as much as $300 \mathrm{ng}$ of dTFIIB, which corresponded to a 180 -fold excess of the amount of TFIIB required for basal transcription. As shown in Figure 5A, the Adh and adenovirus E4 promoters responded differently to excess dTFIIB than the Krüppel and jockey promoters. With the Krüppel and jockey promoters, addition of dTFIIB resulted in an increase in transcription, presumably because TFIIB was a limiting factor in transcription from those promoters. In contrast, excess dTFIIB repressed transcription from the Adh and adenovirus $\mathrm{E} 4$ promoters, which were both minimal promoters containing only the TATA box and RNA start site elements. It thus appears that excess dTFIIB can squelch basal RNA polymerase II transcription and that this squelching occurs only at a subset of promoters.

The underlying basis of TFIIB-mediated squelching was then examined as follows. First, we tested further whether or not the target of TFIIB-mediated repression was a component of the basal transcriptional machinery (e.g., RNA polymerase II) by carrying out experiments in which a "squelchable" promoter (adenovirus E4) and a resistant promoter (Krüppel) were transcribed simultaneously in situ (Fig. 5B). In the same reaction mixture in which E4 transcription is squelched, the Krüppel promoter is unaffected (Fig. 5B, cf. lanes 4 and 8). It therefore appears that the squelched factor is not a basal factor that is required for both $\mathrm{E} 4$ and Krüppel. We then investigated the reversibility of squelching. At an intermediate level of excess dTFIIB (25 ng), the TFIIB-mediated repression of the Adh promoter could be reversed by a two- to threefold increase in the amount of the soluble nuclear fraction (Kamakaka et al. 1991), which was used as a source of basal transcription factors in these experiments (data not shown). Hence, the soluble nuclear fraction contains an activity that is capable of reversing the TFIIB-mediated squelching. Finally, we observed that squelching of the Adh promoter, but not the jockey promoter, occurred when basal transcription was reconstituted with purified RNA polymerase II from Drosophila embryos (Wampler et al. 1990), purified recombinant Drosophila TATA box-binding protein (TBP) (Hoey et al. 1990|, purified recombinant dTFIIB, and partially purified TFIIE/F from Drosophila embryos (Wampler et al. 1990) (data not shown). This result additionally supports the notion that TFIIB-mediated squelching is the result of sequestration of a basal transcription activity rather than a promoter- or enhancer-binding factor. The simplest explanation for these data is that dTFIIB interacts with an essential transcription factor that is required for some, but not all, promoters and that the presence of excess dTFIIB results in sequestering of the essential factor to prevent its assembly into a productive transcription complex (Fig. 5C).

\section{Transcriptional activation by either GAL4-VP16 or Sp1 is not affected by excess dTFIIB}

Recent studies of the mechanism of transcriptional activation by GAL4 derivatives, such as GAL4-AH and GAL4-VP16, have led to the proposal that GAL4 derivatives stimulate transcription by recruiting TFIIB to the preinitiation complex by a mechanism that involves direct interactions between the GAL4 derivative and TFIIB (Lin and Green 1991; Lin et al. 1991; for review, see Hawley 1991; Sharp 1991). Thus, if TFIIB were a limiting component for transcription and GAL4-VP16 functioned to recruit TFIIB to the transcription complex, the addition of excess TFIIB might circumvent the ability of GAL4-VP16 to facilitate transcription. We designate this hypothetical effect as reverse squelching, because it is the inhibition of GAL4-VP16-mediated activation of transcription by a basal factor, as opposed to squelching, which is the inhibition of basal transcription by GAL4VP16.

To investigate the role of TFIIB in transcriptional activation, it was first necessary to test whether or not 


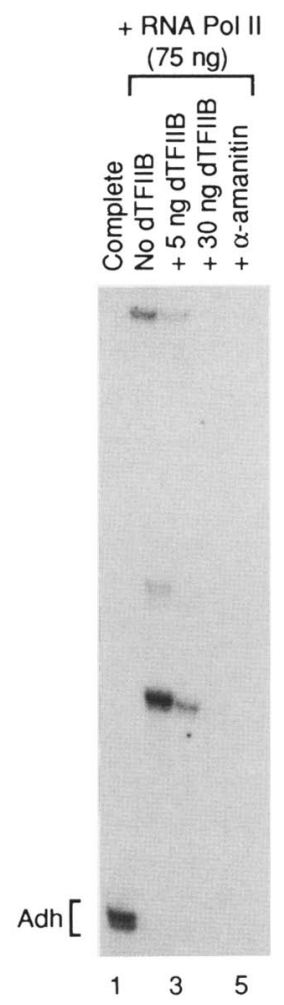

Figure 4. dTFIIB suppresses nonspecific initiation of transcription by RNA polymerase II. Transcription reactions were carried out with the Adh proximal promoter (150 ng of template DNA). (Lane 1) Complete reconstituted transcription reaction; (lane 2) purified RNA polymerase II only (75 ng); (lane 3) RNA polymerase II (75 ng) + dTFIIB (5 ng); (lane 4) RNA polymerase II (75 ng) + dTFIIB (30 ng); (lane 5) RNA polymerase II (75 $\mathrm{ng})+\alpha$-amanitin $(4 \mu \mathrm{g} / \mathrm{ml})$. Transcripts resulting from nonspecific initiation upstream of the normal start site are apparent in lanes 2-4. The primer extension products derived from correct initiation of Adh transcripts are denoted by brackets.

sequence-specific factors were able to activate transcription in a reconstituted transcription reaction with recombinant dTFIIB. We performed transcription reactions that were dependent on the addition of recombinant dTFIIB in the presence or absence of the transcriptional activators GAL4-VP16 or Sp1. As shown in Figure 6, we observed activation of transcription by each of the activator proteins in a binding site-dependent manner. Thus, recombinant dTFIIB was able to function with the remainder of the transcriptional machinery to enable transcriptional activation by either GAL4-VP16 or Spl. These results were also consistent with the findings that transcription reactions containing recombinant hTFIIB were competent for activation by major late transcription factor (MLTF/USF), Spl, and GAL4-VP16 (Ha et al. 1991; Lin et al. 1991).

We then examined the effects of excess dTFIIB on transcriptional activation by either GAL4-VP16 or Sp1. Transcription reactions were performed in the absence or presence of each of the activator proteins with increasing amounts of recombinant dTFIIB (Fig. 7). The highest amount of dTFIIB employed in these experiments, 300 ng, was approximately a 180 -fold excess of the amount of dTFIIB required for a standard transcription reaction. In the presence of varying concentrations of dTFIIB, the magnitude of transcriptional activation by GAL4-VP16 remained relatively constant at approximately three- to fourfold (Fig. 7A), although, as observed previously (Fig. $5 \mathrm{~A})$, there was a general decrease in transcription from the adenovirus E4 promoter as the concentration of dTFIIB was increased. Sp1-mediated activation of transcription was similarly unaffected by addition of dTFIIB to the reactions (Fig. 7B). Hence, excess dTFIIB does not result in reverse squelching of transcriptional activation by sequence-specific factors. The simplest interpretation of these results is that sequence-specific transcription factors do not function to recruit TFIIB to the transcription complex, although, as considered later in the Discussion, more complex models for the role of TFIIB in transcriptional activation can be envisioned.

\section{Discussion}

\section{TFIIB - a simple transcription factor?}

By using purified, recombinant dTFIIB in conjunction with a fractionated in vitro transcription system from Drosophila embryos, we have initiated a functional analysis of the role of TFIIB in basal and regulated transcription by RNA polymerase II. The transcriptionally active form of TFIIB appears to be simple and straightforward. It is a single polypeptide of $\sim 34.5 \mathrm{kD}$, and the E. coli-synthesized protein, which presumably does not possess extensive post-translational modifications, is active for both basal and activated transcription in vitro. Purified, recombinant dTFIIB is fully active at a concentration of one molecule per template DNA. This simplicity, in the midst of the overall complexity of the transcription process, suggests that the study of TFIIB may be a key vantage point for the biochemical analysis of transcription. Identity of $79 \%$ in the primary amino acid sequence of Drosophila and human TFIIB suggests that the entire polypeptide is essential for the transcription process. By using purified, recombinant preparations of TFIIB, we were able to compare the ability of dTFIIB and hTFIIB to function in basal transcription with several promoters. We found that dTFIIB and hTFIIB possessed nearly identical basal transcription activity with the Drosophila Adh, Drosophila jockey, adenovirus E4, and adenovirus major late promoters (Fig. 3 and data not shown), whereas transcription from the Drosophila Krüppel promoter was more efficient with dTFIIB than hTFIIB (Fig. 3). Thus, the relative transcriptional activity of dTFIIB compared with that of hTFIIB is not identical with different promoters, which suggests that there are likely to be variations in the mechanisms by which TFIIB functions in transcription.

\section{Suppression of nonspecific transcription by TFIIB}

Purified RNA polymerase II is capable of nonspecific synthesis of RNA from a DNA template in the absence 


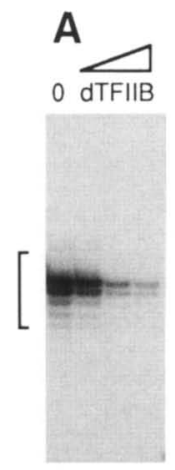

1234

Adh

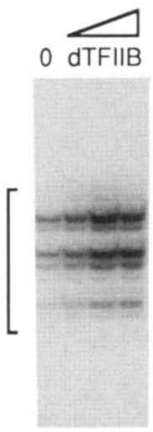

1234

Krüppel

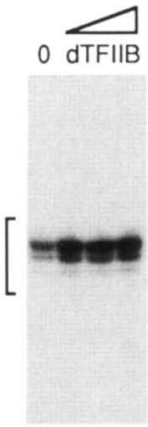

1234

Jockey
Promoter That Does Not Require an Extra Factor an Extra Facto (Adh, AdE4)

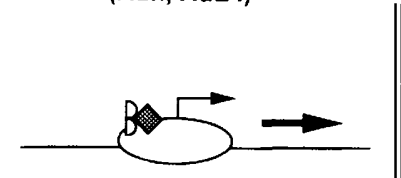
$\lfloor$ Excess TFIIB

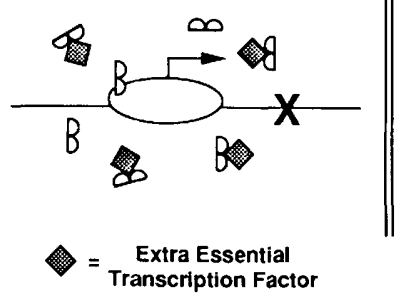
(Kr, Joc)

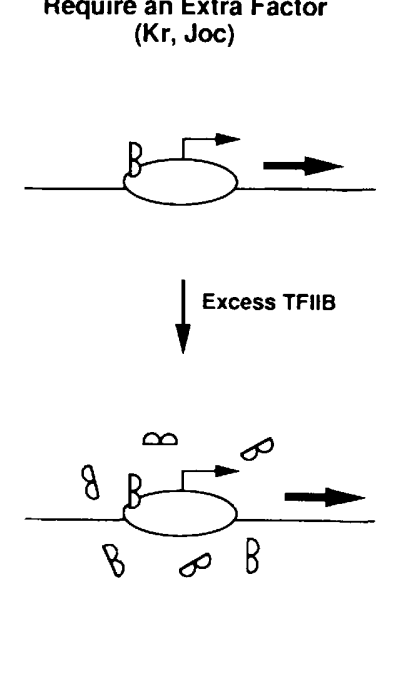

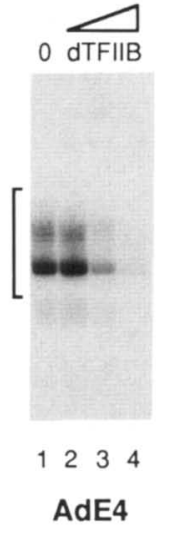

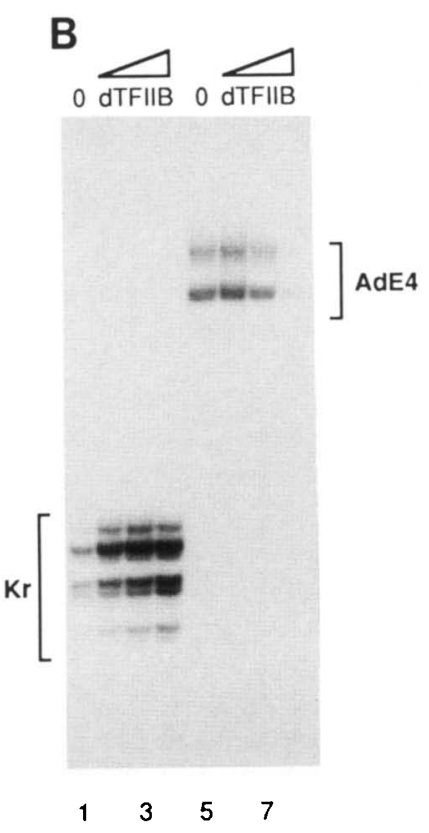

Figure 5. Excess dTFIIB squelches basal transcription in a promoterspecific manner. $(A)$ Transcription reactions were performed with the indicated template DNAs (100 $\mathrm{ng}$ ) by using a soluble nuclear fraction (SNF; $32 \mu \mathrm{g}$ of total protein) from Drosophila embryos (Kamakaka et al. 1991) as the source of the basal transcription factors. The reactions contained either no added dTFIIB (lane 1) or increasing amounts of purified dTFIIB $(7.5,50$, and $300 \mathrm{ng}$, respectively, in lanes 2,3 , and 4$)$. The reverse transcription products are denoted by brackets. $(B)$ Template DNAs (100 ng each) containing either the Krüppel or the adenovirus E4 promoters were transcribed simultaneously in situ under identical conditions as described in $A$. The reactions contained either no added dTFIIB (lanes 1,5$)$ or increasing amounts of dTFIIB $(7.5,50$, and $300 \mathrm{ng}$, respectively, for lanes 2 and 6,3 and 7, 4 and 81 . The reaction products were divided into two equal portions that were separately subjected to reverse transcription analysis with primers complementary to either the Krüppel or E4 transcripts. Thus, the reverse transcription products (which are denoted by brackets) shown in lanes $1 / 5,2 / 6,3 / 7$, and $4 / 8$ each derive from a single transcription reaction. (C) A model for promoter-specific squelching of basal transcription by excess TFIIB. of the general factors, and we have found that this nonspecific initiation of transcription can be suppressed by recombinant dTFIIB (Fig. 4). The TFIIB-mediated repression probably occurs by direct interaction between TFIIB and the polymerase because efficient inhibition of nonspecific transcription is observed at a concentration of one molecule of dTFIIB per $1700 \mathrm{bp}$ of DNA, which renders unlikely possible mechanisms of repression involving extensive DNA binding by TFIIB. It has been shown previously that TFIIF (which is also known as RAP30/74 and $\beta \gamma$ ) can remove RNA polymerase II that is nonspecifically bound to DNA (Conaway and Conaway 1990; Killeen and Greenblatt 1992). In addition, it appears that RAP30, which is the small subunit of TFIIF, can inhibit nonspecific transcription of sonicated calf thymus DNA by RNA polymerase II (Killeen and Greenblatt 1992). It thus appears that both TFIIB and TFIIF can each individ- ually inhibit nonspecific transcription by RNA polymerase, but it is not known whether TFIIB and TFIIF suppress transcription by a similar mechanism. The finding that dTFIIB reduces nonspecific initiation by RNA polymerase II also complements in vivo studies of a yeast homolog of TFIIB, which is encoded by the SUA7 gene (Pinto et al. 1992). In sua7 strains, transcription initiation at normal start sites was decreased while aberrant initiation at other locations was increased. Thus, the requirement for TFIIB in the selection of proper start sites in vivo may be the result of its ability to repress nonspecific initiation of transcription.

\section{GAL4-VP16 does not appear to recruit TFIIB to the transcription complex}

To examine the role of TFIIB in transcriptional activa- 
A

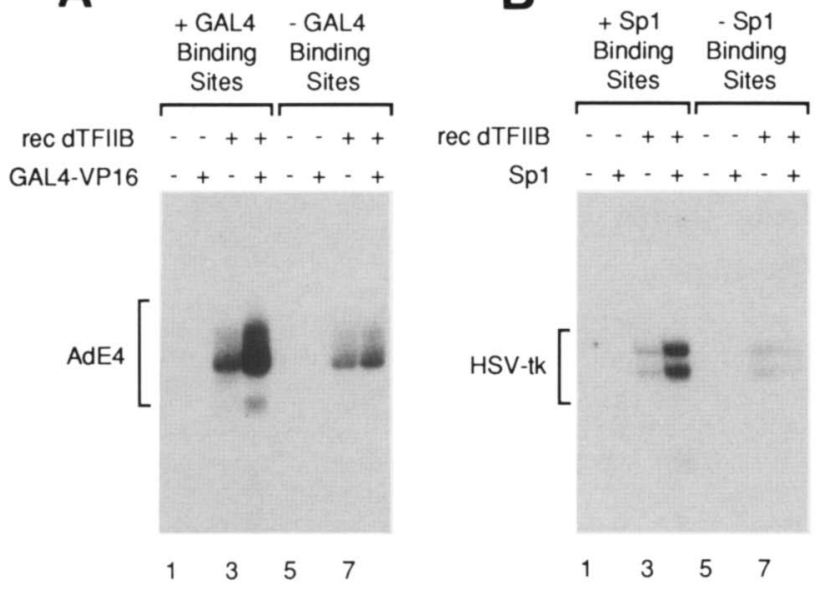

Figure 6. GAL4-VP16 and Spl can mediate activation of transcription in assays reconstituted with recombinant dTFIIB. $(A \mid$ Transcriptional activation by GAL4-VP16. Reactions were carried out with either $\mathrm{pG}_{5} \mathrm{E} 4 \mathrm{~T}$ template DNA, which contains five GAL4-binding sites, or $\mathrm{pG}_{0} \mathrm{E} 4 \mathrm{~T}$, which does not contain GAL4-binding sites (as a control). GAL4-VP16 (0 or $32 \mathrm{ng}$ ) was incubated with template DNAs (100 ng), as indicated. Transcription was initiated by the addition of fractionated Drosophila transcription factors either in the absence of TFIIB or in the presence of purified, recombinant dTFIIB (5 ng). In addition to the basal transcription factors, each of the reactions contained a fraction $(1 \mu \mathrm{g})$ containing an auxiliary activity that is required for GAL4-VP16-mediated activation /G.E. Croston and J.T. Kadonaga, unpubl.). (B) Transcriptional activation by Spl. Reactions were performed with either ptkCAT9, which contains four Spl-binding sites, or p-37tkCAT9, which does not contain Spl binding sites (as a control). Sp1 (0 or $20 \mathrm{ng}$ ) was incubated with the template DNAs ( $100 \mathrm{ng}$, as indicated. Transcription was initiated by the addition of fractionated Drosophila transcription factors either in the absence of TFIIB or in the presence of recombinant dTFIIB (5 ng). The primer extension products of the adenovirus $\mathrm{E} 4$ and herpes simplex virusthymidine kinase (HSV-tk) transcripts are denoted by brackets.

tion, we attempted reverse squelching experiments, in which excess TFIIB might, in principle, inhibit the ability of either GAL4-VP16 or Spl to activate transcription. In these studies we found that a 180 -fold excess of TFIIB did not affect the ability of either GAL4-VP16 or Sp1 to stimulate transcription (Fig. 7). Straightforward interpretation of these data indicates that in contrast to current models, the sequence-specific activator proteins do not function to recruit TFIIB to the transcription complex. An alternative ad hoc explanation of the data is as follows. In the presence of excess TFIIB, GAL4 derivatives may still be required to recruit TFIIB to the transcription complex because the access of TFIIB to the transcription complex may be blocked in the absence of a GAL4 derivative. Such a hypothetical block to binding of TFIIB to the transcription complex is not consistent with the available data on the assembly of the initiation complex, which indicate that TFIIB binds efficiently to a TFIID/ TFIIA/template DNA complex in the absence of se- quence-specific activators (Buratowski et al. 1989; Maldonado et al. 1990; Ha et al. 1991). As noted by Sharp (1991), however, the initial studies of the role of TFIIB in GAL4-AH-mediated activation (Lin and Green 1991) did not consider repression of transcription by nucleosomes or histone $\mathrm{H} 1$, even though it is known that GAL4 derivatives can counteract $\mathrm{Hl}$-mediated transcriptional repression (Croston et al. 1991; Laybourn and Kadonaga 1991) and that the HeLa extracts used in the previous experiments probably contained significant amounts of histone H1 (Croston et al. 1991). Hence, as proposed previously (Sharp 1991), it is possible that histone H1 blocks access of TFIIB to the initiation complex and that GAL4 derivatives function to allow TFIIB assembly into the transcription complex in the presence of $\mathrm{Hl}$.

Another interpretation of the Lin and Green (1991) experiments was proposed by Hawley (1991), who suggested that GAL4-AH may decrease the rate of dissociation of TFIIB from the transcription complexes /which were present on immobilized templates that were subjected to washing with buffer solutions) rather than increase the rate of assembly of TFIIB into the initiation complex. If this interpretation were true, then reaction conditions that are less stringent than the template washing procedure employed by Lin and Green (1991), such as a typical in vitro transcription reaction that is carried out in situ with all of the necessary components, would display a higher basal level of transcription in the absence of the activator and a lower degree of activation

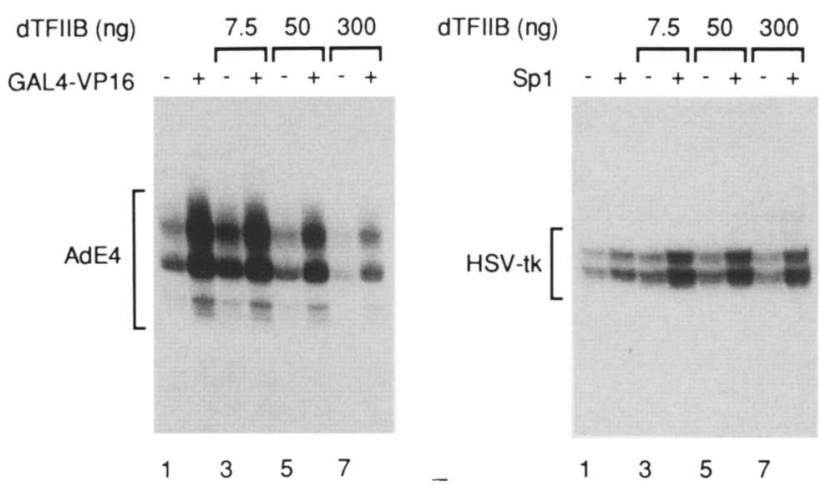

Figure 7. Excess dTFIIB does not affect the magnitude of transcriptional activation by either GAL4-VP16 or Spl. $|A|$ Basal and GAL4-VP16-activated transcription from the adenovirus E4 (AdE4) promoter decrease in parallel in the presence of excess dTFIIB. GAL4-VP16 (0 or $32 \mathrm{ng}$ ) was incubated with $\mathrm{pG}_{5} \mathrm{E} 4 \mathrm{~T}$ template DNA (100 ng), as indicated. Transcription was initiated by the addition of soluble nuclear fraction $(32 \mu \mathrm{g})$ in the presence of recombinant dTFIIB, as noted. $(B)$ Basal and Splactivated transcription from the herpes simplex virus-thymidine kinase (HSV-tk) promoter are not affected by excess dTFIIB. Spl $(0$ or $20 \mathrm{ng}$ ) was incubated with ptkCAT9 template DNA (100 ng), as indicated. Transcription was initiated by the addition of soluble nuclear fraction in the presence of recombinant dTFIIB, as noted. The primer extension products of the adenovirus E4 and HSV-tk transcripts are denoted by brackets. 
by the sequence-specific factor owing to the higher basal transcription. Such an effect was observed by Lin and Green (1991). This hypothesis is also consistent with our observation that excess TFIIB did not affect the magnitude of transcriptional activation by GAL4-VP16 (Fig. 7). It is therefore reasonable to conclude that the present data support the postulate that GAL4 derivatives can stabilize the binding of TFIIB in a transcription complex, but the GAL4 derivatives do not activate transcription by increasing the rate of assembly of TFIIB into the transcription complex.

\section{Promoter-specific squelching of basal transcription by TFIIB}

In our studies of basal transcription, we sought to investigate the effects of excess, purified dTFIIB upon transcription from different promoters. Such squelching experiments have led to the finding that excess dTFIIB represses transcription in a promoter-specific manner (Fig. 5). Although the addition of 30 - to 180 -fold excess of dTFIIB to reactions is probably nonphysiological, the differential response of promoters to such treatment is straightforward. The simplest explanation for these data is that TFIIB interacts with a transcription factor that is essential for the repressed promoters (Adh and adenovirus E4) but not the resistant promoters (jockey and Krüppell, and the addition of excess dTFIIB results in depletion of the extra factor required for the repressed promoters. This model is presented in Figure 5C. Furthermore, the promoters that were squelched by excess TFIIB were minimal promoters containing only the TATA box and RNA start site (Adh, -56 to +383 relative to the start site; adenovirus $\mathrm{E} 4,-38$ to +250 relative to the RNA start site); thus, it is likely that the extra essential factor that was sequestered by dTFIIB is involved in basal transcription. We do not yet know, however, the characteristics that render different promoters to be either squelched or unaffected by excess TFIIB. Notwithstanding, examination of the DNA sequences of the four promoters employed in this study revealed that the squelched promoters (Adh and adenovirus E4) contain consensus TATA boxes, whereas the resistant promoters (jockey and Krüppel) do not possess consensus TATA elements. Whether or not TFIIB-mediated squelching is related to the differences in the transcriptional properties of TATA box-containing versus TATA-less promoters remains to be clarified.

These findings suggest that there may be variable factor requirements for basal transcription of different promoters. Because the generality of the general factors has been presumed rather than proven, the term basal transcription factors may be a more appropriate description of their function. Moreover, it has been found recently that transcription of the immunoglobulin heavy chain gene does not appear to require TFIIE, which is essential for transcription from the adenovirus major late promoter (Parvin et al. 1992). Thus, TFIIE may be a promoter-specific basal transcription factor. The concept of a promoter-specific basal transcription factor is reminis- cent of bacterial $\sigma$-factors and is also aesthetically appealing as a means for providing another dimension of transcriptional regulation beyond that afforded by promoter- and enhancer-binding factors, coactivator/mediators, and chromatin structure.

\section{Materials and methods}

\section{Bacterial strains and plasmids}

E. coli strains HMS174 and BL21 (DE3) were the bacterial hosts used for cloning and expression of dTFIIB in the pET-1 la vector, as described by Studier et al. (1990). Plasmids containing the Adh proximal, jockey, adenovirus E4, and adenovirus major late promoters are as described in Wampler et al. (1990). Plasmid pKr-31/ + 13(119) contains a 44-bp segment of the Drosophila Krüppel promoter from -31 to +13 relative to the major upstream RNA start site inserted into pUC119 (Kerrigan et al. 1991). Transcripts from this promoter were analyzed by using the M13 reverse primer. Plasmids ptkCAT9 and p-37tkCAT9 were described in Courey et al. (1989), and $\mathrm{pG}_{0} \mathrm{E} 4$ and $\mathrm{pG}_{5} \mathrm{E} 4$ were described elsewhere (Lin et al. 1988; Croston et al. 1991).

\section{Preparation of transcription factors.}

The soluble nuclear fraction was prepared by the method of Kamakaka et al. (1991), except that $0.1 \mathrm{M} \mathrm{KCl}$ was used in the nuclei extraction buffer rather than $0.4 \mathrm{M}$ potassium glutamate. Drosophila general transcription factors were fractionated as described previously (Wampler et al. 1990). The TFIIB 0.25-0.5 $\mathrm{M} \mathrm{KCl} \mathrm{S-Sepharose}$ fraction was further purified by successive chromatography on phenyl-Superose (Pharmacia-LKB) and hydroxylapatite (Bio-Rad) resins. TFIIB activity was assayed by primer extension analysis of the in vitro-synthesized RNA. Buffer HEG contains $25 \mathrm{~mm}$ HEPES, $\mathrm{K}^{+}$(pH 7.6), 0.1 mM EDTA, $10 \%$ glycerol, $1.0 \mathrm{mM}$ DTT, and the indicated concentration of ammonium sulfate. Buffer PPEG contains $0.1 \mathrm{~mm}$ EDTA, $15 \%$ glycerol, $1.0 \mathrm{mM}$ DTT, and the indicated amount of potassium phosphate $(\mathrm{pH} 7.6)$. The dialyzed S-Sepharose fraction was adjusted to $1.5 \mathrm{M}$ salt by the addition of $4 \mathrm{M}$ ammonium sulfate ladjusted to $\mathrm{pH} 7.0$ with $\mathrm{NaOH}$ and stored at room temperature), loaded on a phenyl-Superose column equilibrated in $\mathrm{HEG}+1.5 \mathrm{M}$ ammonium sulfate, and eluted with a linear gradient from 1.5 to $0 \mathrm{M}$ ammonium sulfate. Fractions containing TFIIB activity eluted from 0.5 to $0.2 \mathrm{M}$ ammonium sulfate. Insulin $(0.2 \mathrm{mg} / \mathrm{ml}$ final concentration) and NP-40 [0.01\% (vol/ vol) final concentration] were added to each fraction, and the fractions were dialyzed against HEMG-NP $+0.1 \mathrm{M} \mathrm{KCl}$. The dialyzed phenyl-Superose fraction was loaded on hydroxylapatite resin equilibrated in PPEG $+10 \mathrm{~mm}$ potassium phosphate and eluted with salt steps of $0.15,0.4$, and $1.0 \mathrm{M}$ potassium phosphate. This procedure was similar to that of Conaway et al. (1987), with minor modifications. The 0.15-0.4 M HA fraction contained TFIIB activity $(0.01 \mathrm{mg} / \mathrm{ml})$. Insulin $(0.2 \mathrm{mg} / \mathrm{ml}$ final concentration) and NP-40 [0.01\% (vol/vol) final concentration] were added to each fraction, and the fractions were dialyzed against HEMG-NP $+0.1 \mathrm{M} \mathrm{KCl}$. GAL4-VP16 hybrid protein was purified to $\sim 80 \%$ homogeneity as described elsewhere (Chasman et al. 1989; Carey et al. 1990). Transcription factor $\mathrm{Spl}$ was purified from HeLa cells as described in Kadonaga et al. (1987). All protein fractions were frozen in liquid nitrogen and stored at $-100^{\circ} \mathrm{C}$.

\section{Isolation of the CDNA encoding dTFIIB}

Restriction fragments from pIIB1 (Ha et al. 1991) were used to screen a $\lambda \mathrm{gt} 10$ Drosophila embryo cDNA library under low- 
stringency conditions $(30 \%$ formamide, 5\% Denhardt's, $5 \times$ SSPE, $0.1 \%$ SDS). An EcoRI fragment (1587 bp), containing the entire hTFIIB cDNA, and an XhoI fragment ( $687 \mathrm{bp}$ ) were radioactively labeled by oligolabeling (Pharmacia) and hybridized to filters for $24 \mathrm{hr}$ at $42^{\circ} \mathrm{C}$. The filters were washed with $1 \times \mathrm{SST}$, $0.5 \%$ SDS, at $42^{\circ} \mathrm{C}$. A secondary screen was performed with the XhoI fragment and a BsaHI-XhoI (191 bp) amino-terminal fragment as probes. Plaques $\left(3 \times 10^{5}\right)$ were screened to isolate four positive clones. These positive clones were subcloned into the EcoRI site of $\mathrm{M} 13 \mathrm{mp} 18$ for sequencing and mutagenesis. The clones were sequenced by using the Sequenase kit (U.S. Biochemicals).

\section{Expression and purification of recombinant dTFIIB}

An NdeI site was introduced at the first ATG of the dTFIIB cDNA by oligonucleotide-directed in vitro mutagenesis (Amersham). A 1312-bp NdeI-HindIII fragment containing the dTFIIB-coding region was subcloned into pET-1la to yield pETdIIB. Recombinant dTFIIB was expressed in E. coli BL21(DE3) cells and purified on a P11 column (Whatman) according to the method of Ha et al. (1991). dTFIIB was purified further on ceramic hydroxylapatite resin (American International Chemical, Natick, MA) as described in the fractionation section above. The amino terminus of purified dTFIIB was subjected to amino acid sequence analysis to yield the sequence Ala-Ser-Thr-SerArg, which indicated that the amino-terminal methionine residue was removed. We also purified E. coli-synthesized hTFIIB as described by Ha et al. (1991).

\section{In vitro transcription}

Standard in vitro transcription reactions and primer extension analyses of the transcripts were carried out as described by Wampler et al. (1990). Reactions reconstituted with the basal factors contained the following amounts of each fraction: $25 \mathrm{ng}$ of RNA polymerase II; $4 \mu \mathrm{g}$ of TFIID; $0.5 \mu \mathrm{g}$ of TFIIE/F. Transcription reactions containing activator proteins were performed as described by Croston et al. (1991).

\section{Acknowledgments}

We thank Ilho Ha and Danny Reinberg for the generous gift of the human TFIIB clone and sharing information prior to publication. We thank Lucy Lira for expert technical assistance. We are deeply indebted to John Lis (Cornell University, Ithaca, NY) for determining the cytogenetic location of the Drosophila TFIIB gene. We are grateful to Betsy Komives for generous sharing of her oligonucleotide synthesizer; Al Courey for the gift of ptkCAT9 and p-37tkCAT plasmids; Doug Smith for assistance with the computer analysis of the dTFIIB clone; Drs. J. and R. Conaway for the gift of $\alpha$ protein; Matt Williamson for determining the amino-terminal amino acid sequence of recombinant dTFIIB; and Mike Levine, Bruno Zimm, Mike Bulger, Curtis Tyree, Rohinton Kamakaka, Paul Laybourn, and Sumi Paranjape for critical reading of the manuscript. J.T.K. is a Lucille P. Markey Scholar in the Biomedical Sciences. This work was supported in part by grants from the National Institutes of Health (GM 41249) and the Lucille P. Markey Charitable Trust.

The publication costs of this article were defrayed in part by payment of page charges. This article must therefore be hereby marked "advertisement" in accordance with 18 USC section 1734 solely to indicate this fact.

\section{Note added in proof}

The nucleotide sequence of a cDNA encoding dTFIIB has also been reported recently by Yamashita et al. (1992).

\section{References}

Becker, P.B., S.K. Rabindran, and C. Wu. 1991. Heat shock-regulated transcription in vitro from a reconstituted chromatin template. Proc. Natl. Acad. Sci. 884: 109-4113.

Benyajati, C., N. Spoerel, H. Haymerle, and M. Ashburner. 1983. The messenger RNA for alcohol dehydrogenase in Drosophila melanogaster differs in its $5^{\prime}$ end in different developmental stages. Cell 33: 125-133.

Buratowski, S., S. Hahn, L. Guarente, and P.A. Sharp. 1989. Five intermediate complexes in transcription initiation by RNA polymerase II. Cell 56: 549-561.

Carey, M., J. Leatherwood, and M. Ptashne. 1990. A potent GAL4 derivative activates transcription at a distance in vitro. Science 247: 710-712.

Chasman, D.I., J. Leatherwood, M. Carey, M. Ptashne, and R.D. Kornberg. 1989. Activation of yeast polymerase II transcription by herpes VP16 and GAL4 derivatives in vitro. Mol. Cell. Biol. 9: 4746-4749.

Conaway, J.W. and R.C. Conaway. 1990. An RNA polymerase II transcription factor shares functional properties with Escherichia coli $\sigma^{70}$. Science 248: $1550-1553$.

- 1991. Initiation of eukaryotic messenger RNA synthesis. I. Biol. Chem. 266: 17721-17724.

Conaway, J.W., M.W. Bond, and R.C. Conaway. 1987. An RNA polymerase II transcription system from rat liver: Purification of an essential component. I. Biol. Chem. 262: 82938297.

Courey, A.J., D.A. Holtzman, S.P. Jackson, and R. Tjian. 1989. Synergistic activation by the glutamine-rich domains of human transcription factor Sp1. Cell 59: 827-836.

Croston, G.E., L.A. Kerrigan, L.M. Lira, D.R. Marshak, and J.T. Kadonaga. 1991. Sequence-specific antirepression of histone H1-mediated repression of basal RNA polymerase II transcription. Science 251: 643-649.

Dynlacht, B.D., T. Hoey, and R. Tjian. 1991. Isolation of coactivators associated with the TATA-binding protein that mediate transcriptional activation. Cell 66: 563-576.

Felsenfeld, G. 1992. Chromatin as an essential part of the transcriptional mechanism. Nature 355: 219-223.

Flanagan, P.M., R.J. Kelleher III, M.H. Sayre, H. Tschochner, and R.D. Kornberg. 1991. A mediator required for activation of RNA polymerase II transcription in vitro. Nature 350: 436438.

Freedman, L.P., S.K. Yoshinaga, J.N. Vanderbilt, and K.R. Yamamoto. 1989. In vitro transcription enhancement by purified derivatives of the glucocorticoid receptor. Science 245: 298-301.

Gill, G. and M. Ptashne. 1988. Negative effect of the transcriptional activator GAL4. Nature 334: 721-724.

Ha, I., W.S. Lane, and D. Reinberg. 1991. Cloning of a human gene encoding the general transcription initiation factor IIB. Nature 352: 689-695.

Hawley, D. 1991. Transcriptional activation: Enter TFIIB. Trends Biochem. Sci. 16: 317-318.

Heberlein, U., B. England, and R. Tjian. 1985. Characterization of Drosophila transcription factors that activate the tandem promoters of the alcohol dehydrogenase gene. Cell 41: 965977.

Hoey, T., B.D. Dynlacht, M.G. Peterson, B.F. Pugh, and R. Tjian. 1990. Isolation and characterization of the Drosophila gene 
encoding the TATA box binding protein, TFIID. Cell 61: 1179-1186.

Johnson, P.F. and S.L. McKnight. 1989. Eukaryotic transcriptional regulatory proteins. Annu. Rev. Biochem. 58: 799839.

Kadonaga, J.T. 1990. Assembly and disassembly of the Drosophila RNA polymerase II complex during transcription. $J$. Biol. Chem. 265: 2624-2631.

Kadonaga, J.T., K.R. Carner, F.R. Masiarz, and R. Tjian. 1987. Isolation of cDNA encoding transcription factor $\mathrm{Spl}$ and functional analysis of the DNA binding domain. Cell 51: 1079-1090.

Kamakaka, R.T., C.M. Tyree, and J.T. Kadonaga. 1991. Accurate and efficient RNA polymerase II transcription with a soluble nuclear fraction derived from Drosophila embryos. Proc. Natl. Acad. Sci. 88: 1024-1028.

Kerrigan, L.A., G.E. Croston, L.M. Lira, and J.T. Kadonaga. 1991. Sequence-specific transcriptional antirepression of the Drosophila Krüppel gene by the GAGA factor. J. Biol. Chem. 266: 574-582.

Killeen, M.T. and J.F. Greenblatt. 1992. The general transcription factor RAP30 binds to RNA polymerase II and prevents it from binding nonspecifically to DNA. Mol. Cell. Biol. 12: $30-37$

Kornberg, R.D. and Y. Lorch. 1991. Irresistible force meets immovable object: Transcription and the nucleosome. Cell 67: 833-836.

Laybourn, P.J. and J.T. Kadonaga. 1991. Role of nucleosomal cores and histone $\mathrm{Hl}$ in regulation of transcription by RNA polymerase II. Science 254: 238-245.

Lin, Y.-S. and M.R. Green. 1991. Mechanism of action of an acidic transcriptional activator in vitro. Cell 64: 971-981.

Lin, Y.-S., M.F. Carey, M. Ptashne, and M.R. Green. 1988. GAL4 derivatives function alone and synergistically with mammalian activators in vitro. Cell 54: 659-664.

Lin, Y.-S., I. Ha, E. Maldonado, D. Reinberg, and M.R. Green. 1991. Binding of general transcription factor TFIIB to an acidic activating region. Nature 353: 569-571.

Maldonado, E., I. Ha, P. Cortes, L. Weis, and D. Reinberg. 1990. Factors involved in specific transcription by mammalian RNA polymerase II: Role of transcription factors IIA, IID, and IIB during formation of a transcription-competent complex. Mol. Cell. Biol. 10: 6335-6347.

Mitchell, P.J. and R. Tjian. 1989. Transcriptional regulation in mammalian cells by sequence-specific DNA binding proteins. Science 245: 371-378.

Mizrokhi, L.J., S.G. Georgieva, and Y.V. Ilyin. 1988. jockey, a mobile Drosophila element similar to mammalian LINEs, is transcribed from the internal promoter by RNA polymerase II. Cell 54: 685-691.

Moncollin, V., L. Fischer, B. Cavallini, J.-M. Egly, and P. Chambon. 1992. Class II (B) general transcription factor (TFIIB) that binds to the template-committed preinitiation complex is different from general transcription factor BTF3. Proc. Natl. Acad. Sci. 89: 397-401.

Parvin, J.D., H.Th.M. Timmers, and P.A. Sharp. 1992. Promoter specificity of basal transcription factors. Cell 68: 1135-1 144 .

Pinto, I., D.E. Ware, and M. Hampsey. 1992. The yeast SUA7 gene encodes a homolog of human transcription factor TFIIB and is required for normal start site selection in vivo. Cell 68: $977-988$.

Ptashne, M. 1988. How eukaryotic transcriptional activators work. Nature 335: 683-689.

Ptashne, M. and A.A.F. Gann. 1990. Activators and targets. Nature 346: 329-331.

Pugh, B.F. and R. Tjian. 1990. Mechanism of transcriptional activation by Spl: Evidence for coactivators. Cell 61: 11871197.

- 1992. Diverse transcriptional functions of the multisubunit eukaryotic TFIID complex. I. Biol. Chem. 267: 679682.

Reinberg, D. and R.G. Roeder. 1987. Factors involved in specific transcription by mammalian RNA polymerase II: Purification and functional analysis of initiation factors IIB and IIE. I. Biol. Chem. 262: 3310-3321.

Rosenberg, U.B., C. Schröder, A. Preiss, A. Kienlin, S. Côté, I. Riede, and H. Jäckle. 1986. Structural homology of the product of the Drosophila Krüppel gene with Xenopus transcription factor IIIA. Nature 319: 336-339.

Saltzman, A.G. and R. Weinmann. 1989. Promoter specificity and modulation of RNA polymerase II transcription. FASEB 3: 1723-1733.

Sawadogo, M. and A. Sentenac. 1990. RNA polymerase B (II) and general transcription factors. Annu. Rev. Biochem. 59: 711754.

Sharp, P.A. 1991. TFIIB or not TFIIB? Nature 351: 16-18.

Studier, F.W., A.H. Rosenberg, J.J. Dunn, and J.W. Dubendorff. 1990. Use of T7 RNA polymerase to direct expression of cloned genes. Methods Enzymol. 185: 60-89.

Wampler, S.L., C.M. Tyree, and J.T. Kadonaga. 1990. Fractionation of the general RNA polymerase II transcription factors from Drosophila embyros. J. Biol. Chem. 265: 21223-21231.

Wolffe, A.P. 1990. New approaches to chromatin function. New Biol. 2: 211-218.

Yamashita, S., K. Wada, M. Horikoshi, D.-W. Gong, T. Kokubo, K. Hisatake, N. Yokotani, S. Malik, R.G. Roeder, and Y. Nakatani. 1992. Isolation and characterization of a cDNA encoding Drosophila transcription factor TFIIB. Proc. Natl. Acad. Sci. 89: 2839-2843.

Zawel, L. and D. Reinberg. 1992. Initiation of transcription by RNA polymerase II: A multi-step process. Prog. Nucleic Acids Res. Mol. Biol. 45 (in press). 


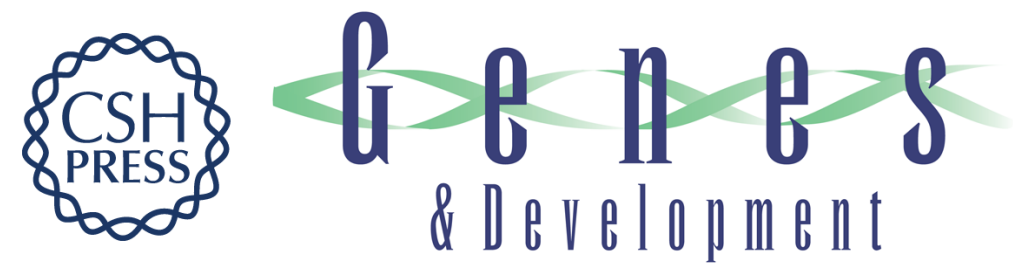

\section{Functional analysis of Drosophila transcription factor IIB.}

S L Wampler and J T Kadonaga

Genes Dev. 1992, 6:

Access the most recent version at doi:10.1101/gad.6.8.1542 $\begin{array}{ll}\text { References } & \begin{array}{l}\text { This article cites } 49 \text { articles, } 19 \text { of which can be accessed free at: } \\ \text { http://genesdev.cshlp.org/content/6/8/1542.full.html\#ref-list-1 }\end{array}\end{array}$

License

Email Alerting Service

Receive free email alerts when new articles cite this article - sign up in the box at the top right corner of the article or click here.

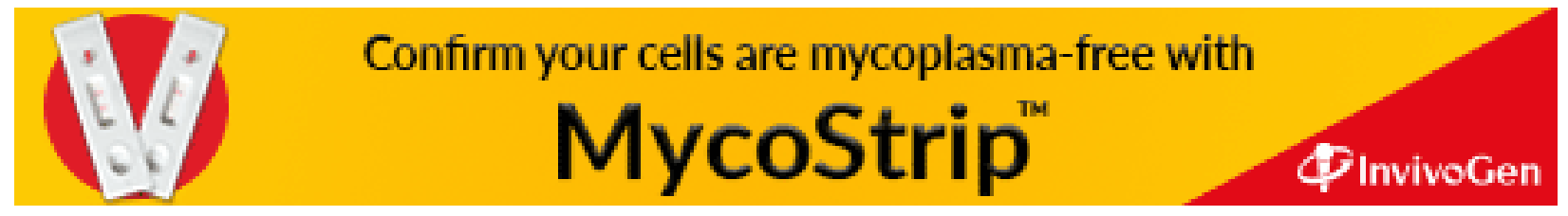

